\title{
How Technology Influences the Therapeutic Process: Evaluation of the Patient-Therapist Relationship in Augmented Reality Exposure Therapy and In Vivo Exposure Therapy
}

\author{
Maja Wrzesien \\ Universitat Politècnica de València, Spain \\ Juana Bretón-López \\ Universidad Jaume I, Castellón, Spain
}

Cristina Botella

Universidad Jaume I, Castellón and Instituto de Salud Carlos III, Spain

Jean-Marie Burkhardt

Institut Français des Sciences et Technologies des Transports, Versailles, France

Mariano Alcañiz

Universitat Politècnica de València, and Instituto de Salud Carlos III, Spain

María Ángeles Pérez-Ara and Antonio Riera del Amo

Universidad Jaume I, Castellón, Spain

Background: New technologies have slowly become a part of psychologists' therapeutic office. However, many therapists still have doubts about the possibility of creating a good

Reprint requests to Maja Wrzesien, Instituto Interuniversitario de Investigación en Bioingeniería y Tecnología Orientada al Ser Humano, Universitat Politècnica de València, I3BH/LabHuman, Cubo Azul - Edif. 8B - Acceso N, Camino de Vera s/n, 46022 Valencia, Spain. E-mail: mwrzesien@labhuman.i3bh.es An extended version is also available online in the table of contents for this issue: http://journals.cambridge.org/jid_BCP 
therapeutic relationship with patients in the presence of technology. Aims: This study evaluates the development of the therapeutic alliance in individuals with small animal phobia disorder who were treated with Augmented Reality Exposure Therapy or In Vivo Exposure Therapy. Method: Twenty-two participants received an intensive session of cognitive behavioural therapy in either a technology-mediated therapeutic context or in a traditional therapeutic context. Results: The results show no significant difference for the therapeutic alliance between two conditions. Conclusions: The results seem to show that technologies such as Augmented Reality do not represent a danger to negatively influence the therapeutic alliance.

Keywords: Therapeutic alliance, Augmented-Reality, in vivo exposure, small animal phobias.

\section{Introduction}

One of the core aspects of the therapeutic relationship is the therapeutic alliance. According to Horvath and Bedi (2002, p. 44), the therapeutic alliance can be expressed as "... the quality and strength of the collaborative relationship between patient and therapist". Despite the growing number of studies on the therapeutic alliance in the traditional face-to-face therapeutic context, only few authors have presented pioneer studies on technology and the therapeutic alliance. For instance, Meyerbröker and Emmelkamp (2008) study the role of therapeutic alliance in Virtual Reality Exposure Therapy. The authors demonstrate that the quality of therapeutic alliance was positively related to the clinical outcome (i.e. reduction in anxiety) in the Virtual Reality Exposure Therapy for the fear of flying. Germain, Marchand, Bouchard, Guay and Drouin (2010) compare the therapeutic alliance in face-to-face vs videoconference treatment of post traumatic stress disorder. The results show that the therapeutic alliance is developed and maintained without significant difference between the two groups. Similarly, Sucala et al. (2012) propose a review of therapeutic relationship in e-therapy. The results show that e-therapy seems to be at least equivalent to face-to-face therapy in terms of therapeutic alliance.

Although numerous empirical studies have demonstrated the clinical effectiveness of the new technologies, many mental health workers still have some concerns regarding the use of technology in clinical practice. Some criticisms concern the space taken up by the hardware in the therapeutic office; the difficulty of creating a therapeutic alliance in the absence of non-verbal communication; and the difficulty of transferring therapeutic interactions to an electronic medium. In a specific case of Augmented Reality (AR) mediated face-to-face therapy, the concerns regarding this particular technology may include the viability and responsiveness of technology, the effect of visual display on the patient-therapist relationship, and the difficulty of anxiety activation with the virtual elements. The aim of this paper is to compare the influence of AR exposure therapy to the traditional face-to-face exposure therapy on the therapeutic alliance.

\section{Method: the case study}

The study compared two types of therapeutic sessions related to the cockroach and spider phobia treatment: the traditional, non-mediated-by-technology In Vivo Exposure Therapy (IVET), and the technology-mediated Augmented Reality Exposure Therapy (ARET, see Juan et al., 2005 for detailed description of the system). The experimental design corresponded 
Table 1. Range, means and standard deviations for WAI overall scores (out of 84), and means and standard deviations for WAI subscales (out of 28) for the two measurement times

Therapeutic sessions

\begin{tabular}{|c|c|c|c|c|}
\hline \multirow[b]{3}{*}{ WAI score } & \multicolumn{4}{|c|}{ Therapeutic sessions } \\
\hline & \multicolumn{2}{|c|}{ In Vivo Exposure Therapy } & \multicolumn{2}{|c|}{$\begin{array}{l}\text { Augmented Reality Exposure } \\
\text { Therapy }\end{array}$} \\
\hline & Pre-session & Post-session & Pre-session & Post-session \\
\hline WAI overall & $76.64(6.74)$ & $80.18(2.82)$ & $76.73(5.62)$ & $78.45(8.61)$ \\
\hline WAI task & $26.45(1.57)$ & $27.45(0.93)$ & $26.18(1.99)$ & $26.36(3.67)$ \\
\hline WAI goal & $26.36(2.94)$ & $26.91(2.02)$ & $26.36(2.11)$ & $26.45(2.54)$ \\
\hline WAI bond & $24.82(3.63)$ & $25.82(2.48)$ & $24.18(2.99)$ & $25.64(3.50)$ \\
\hline
\end{tabular}

to between-subject factorial design, in which the two therapeutic sessions included identical clinical protocol (i.e. same one-session treatment protocol); same objectives; and were located in the same place. The study was approved by the ethical committee from Jaume I University of Castellon, Spain. In total, 22 patients participated in this study $(M=28.18$ years old; $S D=$ 9.42). The patients participated in the diagnostic interview (based on ADIS IV; Di Nardo, Brown and Barlow, 1994), and were randomly assigned to one of two groups. The patients also performed the Behavioural Avoidance Test (BAT; adapted from Öst, 2000) and completed the short version of Working Alliance Inventory (WAI; Tracey and Kokotovic, 1989), after which they received the intensive therapeutic session using one of two treatments. At the end of the session, the evaluation protocol was applied and the patients performed the BAT and completed the WAI scale.

\section{Results}

All the analyses were performed using one-way variance analysis (ANOVA). The analysis of the pretest BAT scores showed no statistically significant differences $[F(1,20)=0.061$, $p=.807]$ between the traditional group $(M=4.64, S D=1.29)$ and the technology-mediated group $(M=4.45, S D=2.07)$ with a statistical power of 0.056 . The analysis of the posttest BAT scores showed no statistically significant differences $[F(1,20)=2.30, p=.145]$ between the traditional group $(M=10.91, S D=1.30)$ and the technology-mediated group $(M=$ $9.91, S D=1.76$ ) with a statistical power of 0.303 . The results of the BAT pretest vs posttest scores within the group comparison showed a statistically significant decline in the severity of avoidance under both conditions $[F(1,19)=68.59, p<.001]$ with a statistical power of 1.00 . No significant difference was observed between the two conditions in this regard $[F(1,19)=$ $1.591, p=.22$, with a statistical power of 0.22 . The analysis of the WAI overall score (see Table 1) demonstrated that the therapeutic alliance is created and maintained as a high quality therapeutic relationship. The statistical analysis did not reveal a significant interaction effect, suggesting that there is no significant difference in therapeutic alliance between the technology-mediated therapeutic session and the traditional therapeutic session $[F(1,19)=$ $0.085, p=.77]$ with power of 0.059 . The analysis of specific WAI dimensions also suggest that there is no significant difference in therapeutic alliance between the two conditions with regard to the goal $[F(1,19)=0.076, p=.79]$, the task $[F(1,19)=1.12, p=.30]$, and the bond $[F(1,19)=0.26, p=.62]$, with a power of $0.058 ; 0.172$; and 0.077 , respectively. 
The results also show a significant increase in quality of therapeutic relationship over time for the WAI overall score $([F(1,19)=5.49, p=.030]$ with a statistical power of 0.604$)$; the WAI task subscale $([F(1,19)=16.68, p<.001]$ with a statistical power of 0.972$)$; and the WAI bond subscale $([F(1,19)=4.60, p=.045]$ with a statistical power of 0.530$)$. The comparison of the specific task, goal, and bond indicators did not show any significant difference between the two groups. The only significant difference was found for item 11 (understanding of the changes that would be good for the patient). The score of this item increased in IVET over time and decreased in ARET over time $[F(1,19)=5.787, p=.026]$ with a statistical power of 0.627 .

\section{Discussion and conclusion}

The objective of this paper was to determine whether technologies such as Augmented Reality can have a negative influence on the therapeutic alliance and, in turn, have an impact on the clinical outcome. The results demonstrated that the therapeutic alliance between the patient and the therapist were the same in both the technology-mediated therapeutic sessions (ARET) and the non-mediated-by-technology therapeutic sessions (IVET). Therefore the results seem to show that technologies such as Augmented Reality do not represent a danger as they do not negatively influence the patient-therapist relationship. The clinical outcome seems to confirm this conclusion.

This study can be improved in several ways. First, although the sample used in this study is relatively high for a study with clinical population, the power to detect the potential differences is low; thus all the conclusions must be confirmed with a larger clinical sample. Second, introduction of a controlled group of therapists with different levels of therapeutic experience might provide a deeper understanding of how technology affects the therapeutic activity according to the therapists' professional experience. Third, there is also a need for additional therapeutic alliance observational methods that complement the understanding of the patient-therapist relationship. This might also diminish the bias due to the use of self-report measures. Moreover, the introduction of the therapeutic alliance scale from the therapists' perspective might bring some interesting additional information. Finally, the therapeutic alliance measures were initially developed in relation to the traditional psychotherapeutic interactions; however, with the introduction of new forms of therapies the therapeutic alliance measures are challenged (Elvins and Green, 2008). Therefore, adapting the WAI scale to Augmented Reality Exposure Therapy should be proposed.

The therapeutic sessions mediated by technologies are slowly moving from the researchers' laboratories to the therapists' offices. We believe that this study is a first step in understanding therapeutic relationship issues in the Augmented Reality-mediated context.

\section{References}

Di Nardo, P. A., Brown, D. A. and Barlow, D. H. (1994). Anxiety Disorders Interview Schedule for DSM-IV: lifetime version (ADIS-IV-L). New York: Graywind Publications.

Elvins, R. and Green, J. (2008). The conceptualization and measurement of therapeutic alliance: an empirical review. Clinical Psychology Review, 28, 1167-1187.

Germain, V., Marchand, A., Bouchard, S., Guay, M. S. and Drouin, S. (2010). Assessment of the Therapeutic Alliance in face-to-face or videoconference treatment for posttraumatic stress disorder. Cyberpsychology, Behavior and Social Networking, 13, 29-35. 
Horvath, A. O. and Bedi, R. P. (2002). The Alliance. In J. C. Norcross (Ed.), Psychotherapy Relationships that Work: therapist contributions and responsiveness to patients. Oxford: Oxford University Press.

Juan, M. C., Alcañiz, M., Monserrat, C., Botella, C., Baños, R. M. and Guerrero, B. (2005). Using Augmented Reality to treat phobias. IEEE Computer Graphics and Applications, 6, 31-37.

Meyerbröker, K. and Emmelkamp, P. M. G. (2008). Therapeutic process in Virtual Reality Exposure Therapy: the role of cognitions and the therapeutic alliance. Journal of Cybertherapy \& Rehabilitation, 1, 247-257.

Öst, L. G. (2000). Rapid treatment of specific phobias. In G. C. L. Davey (Ed.), Phobias: a handbook of theory, research, and treatment (pp. 227-247). New York: Wiley.

Sucala, M., Schnur, J. B., Constantino, J. M., Miller, S. J., Brackmam, E. M. and Montgomery, G. H. (2012). The therapeutic relationship in e-therapy for mental health: a systematic review. Journal of Medical Internet Research, 14, 4, e110.

Tracey, T. J. and Kokotovic, A. M. (1989). Factor structure of the Working Alliance Inventory. Psychological Assessment, 1, 207-210. 